\title{
Usability of Deformable Image Registration for Adaptive Radiotherapy in Head and Neck Cancer and an Automatic Prediction of Replanning
}

\author{
Masao Tanooka1 ${ }^{*}$, Hiroshi Doi ${ }^{2}$, Toshihisa Ishida1, Kazuhiro Kitajima², Tsukasa Wakayama1, \\ Toshiyuki Sakai', Hiroyuki Inoue1, Noriko Kotoura1, Kengo Kosaka², Kazuo Tarutani², \\ Masayuki Fujiwara², Norihiko Kamikonya²
}

${ }^{1}$ Department of Radiological Technology, Hyogo College of Medicine College Hospital, Nishinomiya, Japan

${ }^{2}$ Department of Radiology, Hyogo College of Medicine, Hyogo, Nishinomiya, Japan

Email: ${ }^{\star}$ tanooka@hyo-med.ac.jp

How to cite this paper: Tanooka, M., Doi, H., Ishida, T., Kitajima, K., Wakayama, T., Sakai, T., Inoue, H., Kotoura, N., Kosaka, K., Tarutani, K., Fujiwara, M. and Kamikonya, N. (2017) Usability of Deformable Image Registration for Adaptive Radiotherapy in Head and Neck Cancer and an Automatic Prediction of Replanning. International Journal of Medical Physics, Clinical Engineering and Radiation Oncology, 6, 10-20.

https://doi.org/10.4236/ijmpcero.2017.61002

Received: November 17, 2016

Accepted: January 8, 2017

Published: January 12, 2017

Copyright $\odot 2017$ by authors and Scientific Research Publishing Inc. This work is licensed under the Creative Commons Attribution International License (CC BY 4.0).

http://creativecommons.org/licenses/by/4.0/

\begin{abstract}
Deformable image registration (DIR) has been an important component in adaptive radiotherapy (ART). Our goal was to examine the accuracy of ART using the dice similarity coefficient (DSC) and to determine the optimal timing of replanning. A total of 22 patients who underwent volume modulated arc therapy (VMAT) for head and neck $(\mathrm{H} \& \mathrm{~N})$ cancers were prospectively analyzed. The planning target volume (PTV) was to receive a total of $70 \mathrm{~Gy}$ in 33 fractions. A second planning CT scan (rescan) was performed at the 15th fraction. The DSC was calculated for each structure on both CT scans. The continuous variables to predict the need for replanning were assessed. The optimal cut-off value was determined using receiver operating characteristic (ROC) curve analysis. In the correlation between body weight loss and DSC of each structure, weight loss correlated negatively with DSC of the whole face $\left(r_{s}=-0.45\right)$ and the face surface $\left(r_{s}=-0.51\right)$. Patients who required replanning tended to have experienced rapid weight loss. The threshold DSC was 0.98 and 0.60 in the whole face and the face surface, respectively. Patients who showed low DSC in the whole face and the face surface required replanning at a significantly high rate $(\mathrm{P}<0.05$ and $\mathrm{P}<0.01)$. Weight loss correlated with DSC in both the whole face and the face surface $(\mathrm{P}<0.05$ and $\mathrm{P}<0.05)$. The DSC values in the face predicted the need for replanning. In addition, weight loss tended to correlate with DSC. DIR during ART was found to be a useful tool for replanning.
\end{abstract}

\section{Keywords}

Deformable Image Registration, Adaptive Radiotherapy, Head and Neck 
Cancer, Dice Similarity Coefficient, Volume Modulated Arc Therapy

\section{Introduction}

Modern radiotherapeutic techniques, such as intensity modulated radiation therapy (IMRT) and volumetric modulated arc therapy (VMAT) are considered the standard radiotherapy techniques for the treatment of head and neck squamous cell carcinomas (HNSCC) [1] [2] [3] [4]. These techniques enable delivery of high radiation doses to tumor volumes with reduction of exposure to organs at risk (OARs) [2]. However, geometric and anatomical changes that can occur over a treatment course include tumor and nodal shrinkage, changes in tumor position, and weight loss. This can result in the need to recalculate dosimetry [5] [6] [7].

Various adaptive radiotherapy (ART) techniques have been introduced with the aim of increasing dosimetric accuracy. ART in this context refers to the generation of a new radiotherapy plan based on imaging performed during a patient's treatment course that accounts for the abovementioned changes during treatment. ART with replanning can reduce the doses to OARs and improve patients' quality of life [8] [9] [10] [11] [12]. However, the process of replanning requires additional use of planning equipment and increased staff time.

Several measures for anticipating the need for ART have been described [13] [21]. There are, however, little published data on optimizing the timing of CT rescanning during treatment.

Recently, deformable image registration (DIR) has been become a very important part of ART [19]. For instance, organ contours have been transferred from the planning CT images to the rescan images using DIR methods such as auto-segmentation [22]-[29]. However, the applicability of DIR in IMRT for HNSCC has been less known and poorly established.

We conducted this study to examine the applicability of DIR in ART and to identify the optical timing of the rescan for replanning.

\section{Materials and Methods}

\subsection{Patient Selection and Clinical Characteristics}

Between January 2015 and October 2015, the cases of 22 consecutive patients (17 males and 5 females; median age, 71 years; age range, 44 - 83 years) scheduled to be treated with IMRT for head and neck cancers at our facility were analyzed. This study was approved by the Ethics Committee (approval number: 1631). All patients provided written informed consent to join the prospective cohort study.

Inclusion criteria were histologically-confirmed primary HNSCC; absence of distant metastatic disease; and planned treatment with radical radiotherapy with IMRT technique with or without concurrent chemotherapy. Patients were excluded if they were treated with a three-dimensional conformal radiotherapy 
technique. Patient demographics, tumor characteristics (including pre-treatment size of the dominant node) and treatment details were recorded on standardized forms. Patient, tumor, and treatment characteristics are shown in Table 1.

Table 1. Clinical characteristics of the two groups.

\begin{tabular}{|c|c|c|c|}
\hline Characteristics & Replanning group & Non-replanning group & $\mathrm{P}$-value \\
\hline Number of patients & 11 & 11 & \\
\hline Age (range) & $76(44-81)$ & $68(53-83)$ & 0.41 \\
\hline Sex & & & 0.31 \\
\hline Male & 7 & 10 & \\
\hline Female & 4 & 1 & \\
\hline BMI $\left(\mathrm{kg} / \mathrm{m}^{2}\right)$ & 24.4 & 22.5 & 0.55 \\
\hline (range) & $(17.0-28.66)$ & $(15.9-28.7)$ & \\
\hline Primary tumor site & & & 0.32 \\
\hline Nasopharynx & 1 & 3 & \\
\hline Oropharynx & 3 & 1 & \\
\hline Hypopharynx & 3 & 2 & \\
\hline Larynx & 3 & 1 & \\
\hline Maxillary sinus & 0 & 2 & \\
\hline Ethmoid sinus & 0 & 1 & \\
\hline Nasal cavity & 1 & 0 & \\
\hline Oral tongue & 0 & 1 & \\
\hline Pathological diagnosis & & & 0.33 \\
\hline Squamouscell carcinoma & 11 & 9 & \\
\hline Undifferentiated carcinoma & 0 & 1 & \\
\hline Neuroblastoma & 0 & 1 & \\
\hline Nodal staging & & & 1.00 \\
\hline No & 3 & 3 & \\
\hline $\mathrm{N} 1$ & 3 & 3 & \\
\hline $\mathrm{N} 2$ & 5 & 5 & \\
\hline RT & & & 0.53 \\
\hline Definitive RT & 9 & 10 & \\
\hline Postoperative RT & 2 & 1 & \\
\hline Prescription doses of RT & $70(60-70) \mathrm{Gy}$ & $70(60-70) \mathrm{Gy}$ & 0.17 \\
\hline 70 Gy & 9 & 7 & \\
\hline $66 \mathrm{~Gy}$ & 0 & 3 & \\
\hline 60 Gy & 2 & 1 & \\
\hline Chemotherapy & & & 0.11 \\
\hline Carboplatin & 1 & 3 & \\
\hline Docetaxel & 3 & 1 & \\
\hline Cisplatin & 1 & 3 & \\
\hline Cetuximab & 4 & 0 & \\
\hline None & 2 & 4 & \\
\hline \multicolumn{4}{|c|}{ Timing of the rescan from the initiation of RT } \\
\hline Fractions & $15(14-19)$ & $15(13-18)$ & 0.69 \\
\hline Days & $21(17-30)$ & $20(17-27)$ & 0.91 \\
\hline $\mathrm{BMI}$ at rescan $\left(\mathrm{kg} / \mathrm{m}^{2}\right)$ & 23.1 & 21.3 & 0.78 \\
\hline (range) & $(16.5-27.9)$ & $(14.4-27.3)$ & \\
\hline \multicolumn{4}{|c|}{ Weight loss from the initiation of RT to the rescan (\%) } \\
\hline (range) & $5.4(1.7-11.2)$ & $4.1(-3.2-9.2)$ & 0.19 \\
\hline
\end{tabular}

Abbreviations: BMI, body mass index; RT, radiotherapy; CT, computed tomography. 


\subsection{Radiotherapy}

All the patients were placed in the supine position and scanned using an Aquilion LB computed tomography (CT) unit (Toshiba, Ohtawara, Japan). The CT dataset was transferred to an XiO treatment planning system (Elekta, Stockholm, Sweden) or MIM Maestro (MIM Software, Inc., Cleveland OH, USA) to outline the volumes of interest (VOIs), and then transferred to a commercial Monte Carlo-based treatment-planning system (TPS), Monaco 2.03 (Elekta, St. Louis, MO, USA).

Intravenous contrast was used for CT scanning in patients with lymphadenopathy. All patients had a positron emission tomography (PET)/CT fused with the planning CT for volume definition. Magnetic resonance imaging (MRI) scans were fused as needed with the planning CT scan to aid in target delineation except for patients receiving adjuvant radiotherapy following surgery. Target volumes were contoured according to the department's standard protocol.

Radiotherapy was designed for VMAT by using a simultaneous integrated boost IMRT (SIB-IMRT) technique in all eligible patients. In patients receiving radiotherapy as their initial treatment, planning target volumes (PTV) were designed as follows: PTV70 received 70 Gy in $2.12 \mathrm{~Gy} /$ fraction for gross tumor and metastatic nodes, PTV59.4 received $59.4 \mathrm{~Gy}$ in $1.8 \mathrm{~Gy} /$ fraction for clinical tumor volumes (CTV) that were considered high-risk, and PTV54 received $54 \mathrm{~Gy}$ in $1.64 \mathrm{~Gy} /$ fraction for CTVs that were considered as high-risk over 33 days. Treatment regimens and prescription doses are shown in Table 1 . The planned radiotherapy was delivered using an Elekta Synergy device (Elekta, Crawley, UK). In addition, daily image guided radiation therapy (IGRT) was performed with cone beam CT. Clinical findings and body weight were recorded once a week. A single radiation oncologist saw all patients and checked and drew all structures. The decision of the need for replanning was made with no consideration of the following analysis.

\subsection{Imaging Analysis and Deformable Image Registration}

A rescan was planned to be performed at fraction 15 prior to treatment commencement. DIR was performed using a commercial software package, MIM Maestro (MIM Soft-ware, Inc., Cleveland, OH, USA). The process started with a rigid coregistration of the planning CT and the rescan images. Then, the DIR and adaptive contouring module in MIM Maestro (a proprietary intensity-based algorithm) deformed the structures on the planning images to match the rescan images. PTV and OARs including the spinal cord, brain stem, and body outline were analyzed. In addition, the face was analyzed with regard to the volume (whole face) and the face surface (the top $3 \mathrm{~mm}$ of skin). The dice similarity coefficient (DSC) was used to evaluate for similarity between the planning CT (A) and the rescan (B), using a scale that ranges from 0 for no correspondence between the images to 1 for complete correspondence. The DSC is defined as follows: 


$$
D S C=\frac{2 \cdot A \bigcap B}{|A|+|B|}
$$

\subsection{Statistical Analysis}

We analyzed the weight loss rate and DSC in patients who required replanning (the replanning group) and in patients who did not require replanning (the non-replanning group\}. The data are expressed as medians with the ranges in parentheses, unless otherwise indicated. In regards to the association between the two groups, continuous variables and incidence of patients, the trend for incidence was assessed using the Mann-Whitney U-test, the Fisher's exact test and Chi-square test for trend, respectively. The optimal cut-off value was determined using receiver operating characteristic (ROC) curve analysis. All statistical analyses were performed using GraphPad Prism version 6.0b (GraphPad Software Inc., San Diego, CA, USA). A P value $<0.05$ was considered statistically significant.

\section{Results}

The clinical characteristics of the two groups are shown in Table 1. No significant differences were observed in the clinical parameters between the two groups. The changes in body weight are shown in Figure 1. Not surprisingly, patients' body weights decreased with time. The weight loss rate at rescan is shown in Figure 2. No significant differences were observed at rescan. However, the replanning group had weight loss over the duration of radiotherapy and also at rescan. Table 2 shows the DSC of each structure in the replanning and nonreplanning groups. Patients who required replanning showed significantly smaller DSC values in all analyzed structures except for the spinal cord than those who did not require replanning. Based on this data, we focused on the face surface and whole face in the following analysis. The correlation between weight

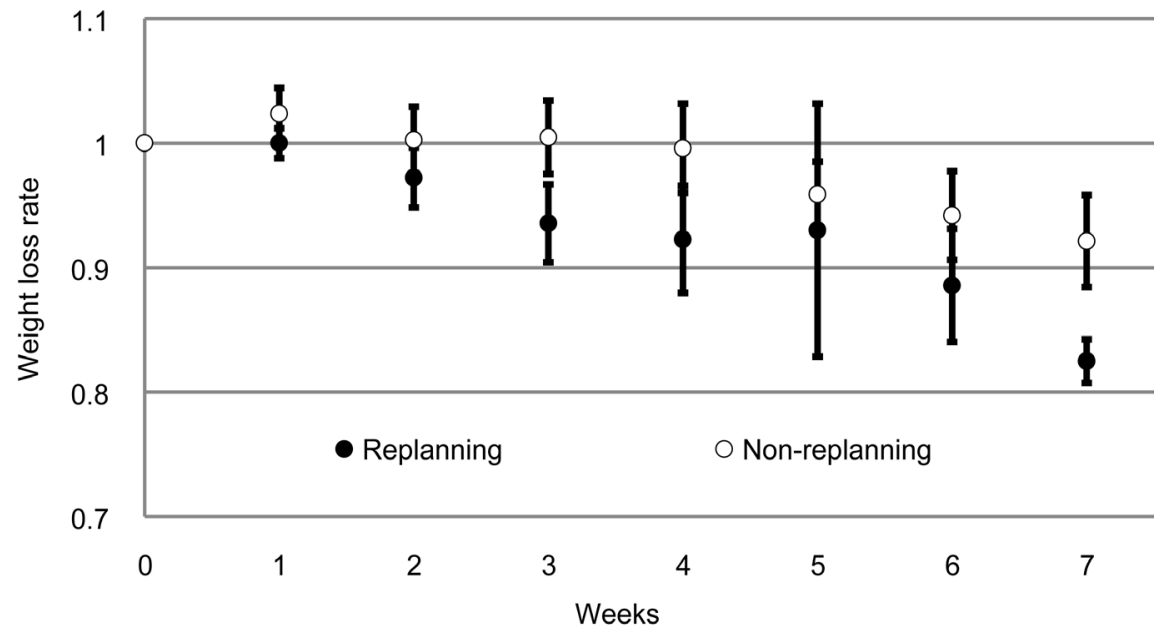

Figure 1. Weight changes in the course of radiotherapy. Patients who needed replanning showed greater weight loss than those who did not. Each point of the two groups is the mean weight loss, and error bars represent one standard deviation. 


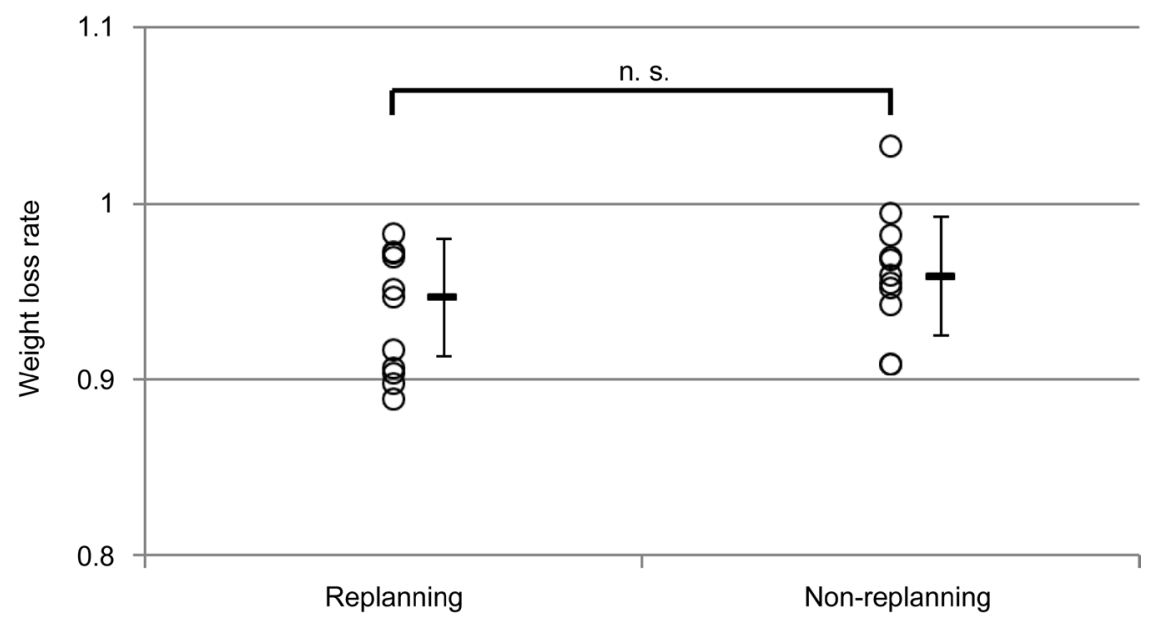

Figure 2. Comparison of weight loss rate between the two groups at rescan. A rescan was planned to be performed at fraction 15 prior to treatment commencement. The weight loss was $5.4 \%(1.7 \%-11.2 \%)$ in the replanning group and $4.1 \%(-3.2 \%-9.2 \%)$ in the non-replanning group. No significant differences were observed between the two groups $(\mathrm{P}=0.19)$. Median value and standard deviation are placed on the right. n.s.: not significant.

Table 2. DSC of each structure in the replanning and non-replanning groups.

\begin{tabular}{cccc}
\hline & Replanning group & Non-replanning group & P-value \\
\hline PTV & 0.856 & 0.928 & $<0.05$ \\
Body & 0.964 & 0.976 & 0.04 \\
Brainstem & 0.855 & 0.941 & 0.05 \\
Spinal cord & 0.747 & 0.841 & 0.07 \\
Face surface & 0.420 & 0.655 & $<0.001$ \\
Whole face & 0.960 & 0.979 & $<0.01$ \\
\hline
\end{tabular}

Abbreviations: DSC, dice similarity coefficient; PTV, planning target volume.

loss and DSC was analyzed (Figure 3). There was a significantly negative correlation between the weight loss and DSC in the face surface and whole face. Using the cut-off values of 0.60 and 0.98 for the face surface and whole face, respectively, the replanning group included significantly more patients who developed low DSC values (Figure 4).

\section{Discussion}

IMRT is a well-established standard technique of radiotherapy for HNSCC. SIB-IMRT has the advantages of better target conformity, lower dose to critical structures, moderate treatment acceleration with reduced total treatment time, and the option of dose escalation in the gross tumor volume. In addition, SIB-IMRT can be delivered with a shorter process and save resources compared with conventional sequential boost IMRT. High accuracy is necessary and the same status is ideally required through the treatment duration. However, patients' physical condition can dramatically change over the treatment duration 

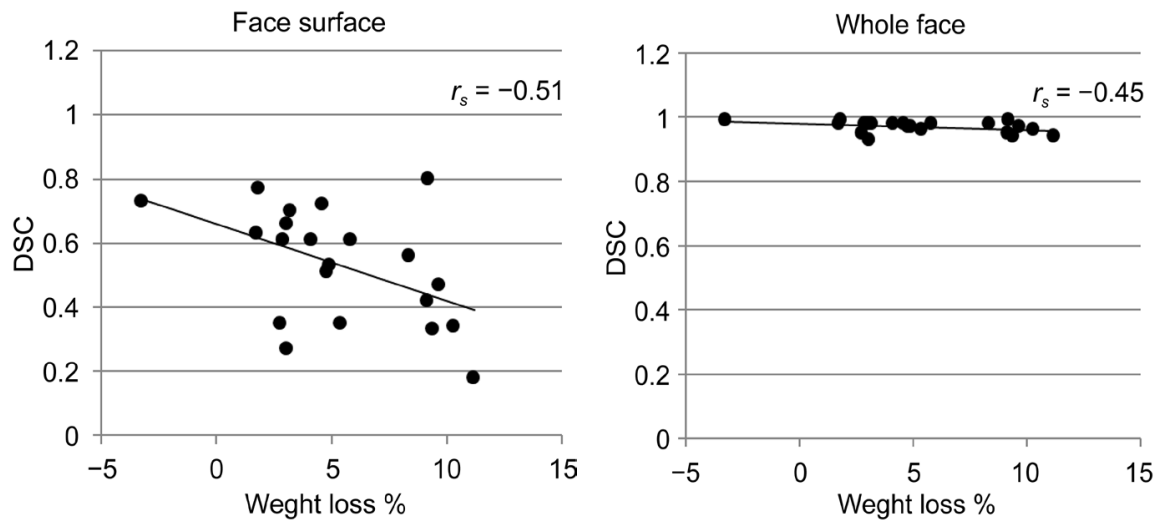

Figure 3. Relationship between weight loss and DSC. Significant negative correlations were observed between the weight loss and DSC in face surface $\left(r_{s}=-0.51, \mathrm{P}<0.05\right)$ and whole face $\left(r_{s}=-0.45, \mathrm{P}<0.05\right)$.
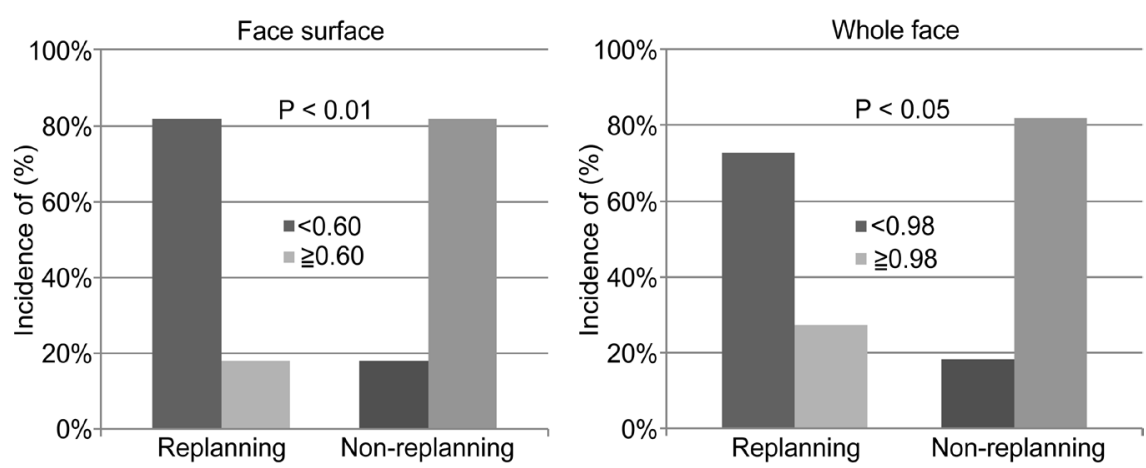

Figure 4. Analysis using the cut-off values in two structures. Compared with the nonreplanning group, the replanning group included significantly more patients who developed low DSC values in face surface and whole face $(\mathrm{P}<0.01, \mathrm{P}<0.05$, respectively).

because of weight loss and tumor shrinkage. The considerable anatomical changes during the course of radiotherapy may lead to suboptimal dosimetry. Several factors have been reported to predict the need for replanning [8] [9] [13] [14] [15]. However, the optimal timing for replanning is poorly understood.

In the present study, a positive correlation between weight loss and the need for replanning was observed but statistical significance was not reached. Greater weight loss significantly decreased DSC in targets and also OARs. Additionally, patients who needed replanning showed significantly lower DSC values in PTVs including the whole body, whole face, and face surface than those who did not. In addition, ROC analysis revealed the cut-off values of 0.60 and 0.98 for the face surface and whole face, respectively. Using our cut-off values, high DSC values in the face surface and whole face can predict the need for replanning. Therefore, we suggest calculating the DSC of the face surface and whole face in the rescan to assess the need for replanning without manual modifications and subjective evaluation.

The face surface and whole face are relatively large structures whose sizes can improve the sensitivity of replanning. DSC calculations from the rescan can save human resources and time. Greater weight loss predicts the requirement of the 
modification of structures after auto-segmentation using DIR and that of replanning. We showed that weight loss tended to correlate DSC in a prospective study setting. In this study, the degree of weight loss had insufficiently strong correlation with DSC and the need for replanning. A small sample size might have affected the statistical results. In addition, the head and neck area contain relatively small volumes of fat. It has been reported that body mass index affected the incidence of toxicity in prostate cancer patients [30]. In this study, the clinical findings of toxicity were not mentioned. We expect that further prospective clinical studies with larger numbers of patient would reveal the predictive factors more clearly and the effects of ART and weight loss on anti-tumor effect and toxicity.

Brown et al. recently reported a predictive model and the decision tree for ART [13]. However, several parameters such as an initial weight $>100 \mathrm{~kg}$ are not applicable to an Asian population and complicate its clinical use. We showed that weight loss could be a simple and valuable parameter to predict the requirement of replanning with manual contouring. Auto-contouring with DIR is a useful tool for ART in clinical practice and can potentially save human resources and time. However, large anatomical changes can cause significant changes in the structures in excess of the adaptivity of the software, leading to errors. Our results indicate weight loss can predict not only the need for replanning but also manually contouring. A further study is needed to show the threshold of weight loss during the duration of radiotherapy and the optimal timing of the rescan for replanning.

We acknowledge that our study has several limitations. Eligible patients had variations in their background characteristics such as differences in primary tumors and delivered radiotherapeutic doses. However, our data was collected prospectively and the focus of our interest was the adequacy of ART with DIR and if weight loss can play a significant role in ART dosimetry. In addition, a single radiation oncologist drew all structures, evaluated the images after DIR, and made decisions on the need for replanning. We therefore believe our data are reliable. In addition, there was no significant difference between weight loss and the requirement for replanning. The sample size was small in this prospective study. However, a tendency of a positive relationship between weight loss and the requirement of replanning was observed. Therefore, based on these data, further prospective data collection might reveal a significant difference and enable the prediction of replanning. Imaging data collected for IGRT during treatment, such as cone beam CT, could also be a useful tool for prediction of replanning [31] [32].

\section{Conclusion}

DSC that was automatically calculated using CT and DIR technique was a simple and useful parameter to predict the needs for manual contouring and replanning. The cut-off values of DSC were 0.60 and 0.98 for the face surface and whole face, respectively. 


\section{Acknowledgements}

We would like to thank DMC Corp. (www.dmed.co.jp) for English language editing. This work was supported by a Grant-in-Aid for Young Scientists (B) Grant Number 25861134.

\section{References}

[1] Pfister, D.G., Spencer, S., Brizel, D.M., Burtness, B., Busse, P.M. and Caudell, J. (2015) Head and Neck Cancers, Version 1.2015. Journal of the National Comprehensive Cancer Network, 7, 847-856. http://www.jnccn.org/content/13/7/847.short

[2] Kam, M.K., Leung, S.F., Zee, B., Chau, R.M., Suen, J.J. and Mo, F. (2007) Prospective Randomized Study of Intensity-Modulated Radiotherapy on Salivary Gland Function in Early-Stage Nasopharyngeal Carcinoma Patients. Journal of Clinical Oncology, 25, 4873-4879. https://doi.org/10.1200/JCO.2007.11.5501

[3] Johnston, M., Clifford, S., Bromley, R., Back, M., Oliver, L. and Eade, T. (2011) Volumetric-Modulated Arc Therapy in Head and Neck Radiotherapy: A Planning Comparison Using Simultaneous Integrated Boost for Nasopharynx and Oropharynx Carcinoma. Clinical Oncology, 23, 503-511. https://doi.org/10.1016/j.clon.2011.02.002

[4] Songthong, A.P., Kannarunimit, D., Chakkabat, C. and Lertbutsayanukul, C. (2015) A Randomized Phase II/III Study of Adverse Events between Sequential (SEQ) Versus Simultaneous Integrated Boost (SIB) Intensity Modulated Radiation Therapy (IMRT) in Nasopharyngeal Carcinoma; Preliminary Result on Acute Adverse Events. Radiation Oncology, 10, 166. https://doi.org/10.1186/s13014-015-0472-y

[5] Chen, C., Fei, Z., Chen, L., Bai, P., Lin, X. and Pan, J. (2014) Will Weight Loss Cause Significant Dosimetric Changes of Target Volumes And Organs at Risk in Nasopharyngeal Carcinoma Treated with Intensity-Modulated Radiation Therapy? Medical Dosimetry, 39, 34-37. https://doi.org/10.1016/j.meddos.2013.09.002

[6] Beltran, M., Ramos, M., Rovira, J.J., Perez-Hoyos, S. and Sancho, M. (2012) Dose Variations in Tumor Volumes and Organs at Risk during IMRT for Head-and-Neck Cancer. Journal of Applied Clinical Medical Physics, 13, 3723.

[7] Bhide, S.A., Davies, M., Burke, K., McNair, H.A., Hansen, V. and Barbachano, Y. (2010) Weekly Volume and Dosimetric Changes during Chemoradiotherapy with Intensity-Modulated Radiation Therapy for Head and Neck Cancer: A Prospective Observational Study. International Journal of Radiation Oncology ${ }^{\star}$ Biology ${ }^{\star}$ Physics, 76, 1360-1368. https://doi.org/10.1016/j.ijrobp.2009.04.005

[8] Hansen, E.K., Bucci, M.K., Quivey, J.M., Weinberg, V. and Xia, P. (2006) Repeat CT Imaging and Replanning during the Course of IMRT for Head-and-Neck Cancer. International Journal of Radiation Oncology ${ }^{\star}$ Biology ${ }^{\star}$ Physics, 64, 355-362. https://doi.org/10.1016/j.ijrobp.2005.07.957

[9] Yang, H., Hu, W., Wang, W., Chen, P., Ding, W. and Luo, W. (2013) Replanning during Intensity Modulated Radiation Therapy Improved Quality of Life in Patients with Nasopharyngeal Carcinoma. International Journal of Radiation Oncology ${ }^{\star} \mathrm{Bi}$ ology $^{\star}$ Physics, 85, e47-e54. https://doi.org/10.1016/j.ijrobp.2012.09.033

[10] Castelli, J., Simon, A., Louvel, G., Henry, O., Chajon, E. and Nassef, M. (2015) Impact of Head and Neck Cancer Adaptive Radiotherapy to Spare the Parotid Glands and Decrease the Risk of Xerostomia. Radiation Oncology, 10, 1. https://doi.org/10.1186/s13014-014-0318-Z

[11] Bhandari, V., Patel, P., Gurjar, O.P. and Gupta, K.L. (2014) Impact of Repeat Computerized Tomography Replans in the Radiation Therapy of Head and Neck Can- 
cers. Journal of Medical Physics, 39, 164-168. https://doi.org/10.4103/0971-6203.139005

[12] Ahn, P.H., Chen, C.C., Ahn, A.I., Hong, L., Scripes, P.G. and Shen, J. (2011) Adaptive Planning in Intensity-Modulated Radiation Therapy for Head and Neck Cancers: Single-Institution Experience and Clinical Implications. International Journal of Radiation Oncology, Biology, Physics, 80, 677-685. https://doi.org/10.1016/j.ijrobp.2010.03.014

[13] Brown, E., Owen, R., Harden, F., Mengersen, K., Oestreich, K. and Houghton, W. (2015) Predicting the Need for Adaptive Radiotherapy in Head and Neck Cancer. Radiotherapy and Oncology, 116, 57-63. https://doi.org/10.1016/j.radonc.2015.06.025

[14] Fiorentino, A., Cozzolino, M., Caivano, R., Pedicini, P., Oliviero, C. and Chiumento, C. (2014) Head and Neck Intensity Modulated Radiotherapy Parotid Glands: Time of Re-Planning. La Radiologia Medica, 119, 201-207. https://doi.org/10.1007/s11547-013-0326-3

[15] Markus, S., Kristina, G., Jürgen, D., Rolf, B. and Eva, M.S. (2014) The Frequency of Re-Planning and Its Variability Dependent on the Modification of the Re-Planning Criteria and IGRT Correction Strategy in Head and Neck IMRT. Radiation Oncology, 9, 175. https://doi.org/10.1186/1748-717X-9-175

[16] Ding, G.X., Duggan, D.M., Coffey, C.W., Deeley, M., Hallahan, D.E., Cmelak, A. and Malcolm, A. (2007) A Study on Adaptive IMRT Treatment Planning Using kV Cone-Beam CT. Radiotherapy and Oncology, 85, 116-125. https://doi.org/10.1016/j.radonc.2007.06.015

[17] Fiorentino, A., Cozzolino, M., Caivano, R., Pedicini, P., Chiumento, C. and Oliviero, C. (2013) Cone-Beam Computed Tomography Dose Monitoring during Intensity-Modulated Radiotherapy in Head and Neck Cancer: Parotid Glands. Clinical and Translational Oncology, 15, 412-415. https://doi.org/10.1007/s12094-012-0946-4

[18] Hu, C.C., Huang, W.T., Tsai, C.L., Wu, J.K., Chao, H.L. and Huang, G.M. (2011) Practically Acquired and Modified Cone-Beam Computed Tomography Images for Accurate Dose Calculation in Head and Neck Cancer. Strahlentherapie und Onkologie, 187, 633-644. https://doi.org/10.1007/s00066-011-2247-1

[19] Elstrøm, U.V., Wysocka, B.A., Muren, L.P., Petersen, J.B. and Grau, C. (2010) Daily $\mathrm{kV}$ Cone-Beam CT and Deformable Image Registration as a Method for Studying Dosimetric Consequences of Anatomic Changes in Adaptive IMRT of Head and Neck Cancer. Acta Oncologica, 49, 1101-1108. https://doi.org/10.3109/0284186X.2010.500304

[20] Wu, B., Ricchetti, F., Sanguineti, G., Kazhdan, M., Simari, P. and Jacques, R. (2011) Data-Driven Approach to Generating Achievable Dose-Volume Histogram Objectives in Intensity-Modulated Radiotherapy Planning. International Journal of Radiation Oncology, Biology, Physics, 79, 1241-1247. https://doi.org/10.1016/j.ijrobp.2010.05.026

[21] Lee, L., Le, Q.T. and Xing, L. (2008) Retrospective IMRT Dose Reconstruction Based on Cone-Beam CT and MLC Log-File. International Journal of Radiation Oncology, Biology, Physics, 70, 634-644. https://doi.org/10.1016/j.ijrobp.2007.09.054

[22] Hilts, M., Batchelar, D., Rose, J. and Crook, J. (2015) Deformable Image Registration for Defining the Postimplant Seroma in Permanent Breast Seed Implant Brachytherapy. Brachytherapy, 14, 409-418.

https://doi.org/10.1016/j.brachy.2014.11.003 
[23] Andersen, E.S., Noe, K.Ø., Sørensen, T.S., Nielsen, S.K., Fokdal, L. and Paludan, M. (2013) Simple DVH Parameter Addition as Compared to Deformable Registration for Bladder Dose Accumulation in Cervix Cancer Brachytherapy. Radiotherapy and Oncology, 107, 52-57. https://doi.org/10.1016/j.radonc.2013.01.013

[24] Christensen, G.E., Carlson, B., Chao, K.C., Yin, P., Grigsby, P.W. and Nguyen, K. (2001) Image-Based Dose Planning of Intracavitary Brachytherapy: Registration of Serial-Imaging Studies Using Deformable Anatomic Templates. International Journal of Radiation Oncology, Biology, Physics, 51, 227-243.

https://doi.org/10.1016/S0360-3016(01)01667-4

[25] Kim, H., Huq, M.S., Houser, C., Beriwal, S. and Michalski, D. (2014) Mapping of Dose Distribution from IMRT onto MRI-Guided High Dose Rate Brachytherapy Using Deformable Image Registration for Cervical Cancer Treatments: Preliminary Study with Commercially Available Software. Journal of Contemporary Brachytherapy, 6, 178-184. https://doi.org/10.5114/jcb.2014.43240

[26] Sebastia, S., Ignacio, A., Marimar, S., Roberto, B., Santiago, M.H. and Meritxell, A. (2012) Dose Accumulation during Vaginal Cuff Brachytherapy Based on Rigid/ Deformable Registration vs. Single Plan Addition. Brachytherapy, 13, 343-351.

[27] Hu, Y., Ahmed, H.U., Taylor, Z., Allen, C., Emberton, M., Hawkes, D. and Barratt, D. (2012) MR to Ultrasound Registration for Image-Guided Prostate Interventions. Medical Image Analysis, 16, 687-703. https://doi.org/10.1016/j.media.2010.11.003

[28] Pursley, J., Risholm, P., Fedorov, A., Tuncali, K., Fennessy, F.M. and Wells, W. (2012) A Bayesian Nonrigid Registration Method to Enhance Intraoperative Target Definition in Image-Guided Prostate Procedures through Uncertainty Characterization. Medical Physics, 39, 6858-6867. https://doi.org/10.1118/1.4760992

[29] Chen, T., Nie, K., Narra, V., Zou, J., Zhang, M. and Khan, A. (2014) Dosimetric Analysis on Breast Brachytherapy Based on Deformable Image Registration. Medical Physics, 41, 218-218. https://doi.org/10.1118/1.4888317

[30] Ishimaru, F., Tanooka, M., Inoue, H., Odawara, S., Takada, Y. and Niwa, Y. (2014) Body Mass Index Can Affect Gastrointestinal and Genitourinary Toxicity in $\mathrm{Pa}$ tients with Prostate Cancer Treated with External Beam Radiation Therapy. Oncology Letters, 7, 209-214.

[31] Ciardo, D., Alterio, D., Jereczek-Fossa, B.A., Riboldi, M., Zerini, D. and Santoro, L. (2015) Set-Up Errors in Head and Neck Cancer Patients Treated with Intensity Modulated Radiation Therapy: Quantitative Comparison between Three-Dimensional Cone-Beam CT and Two-Dimensional Kilovoltage Images. Physica Medica, 31, 1015-1021. https://doi.org/10.1016/j.ejmp.2015.08.004

[32] Qi, X.S., Santhanam, A., Neylon, J., Min, Y., Armstrong, T. and Sheng, K. (2015) Near Real-Time Assessment of Anatomic and Dosimetric Variations for Head and Neck Radiation Therapy via Graphics Processing Unit-Based Dose Deformation Framework. International Journal of Radiation Oncology, Biology, Physics, 92, 415422. https://doi.org/10.1016/j.ijrobp.2015.01.033 
Submit or recommend next manuscript to SCIRP and we will provide best service for you:

Accepting pre-submission inquiries through Email, Facebook, LinkedIn, Twitter, etc. A wide selection of journals (inclusive of 9 subjects, more than 200 journals)

Providing 24-hour high-quality service

User-friendly online submission system

Fair and swift peer-review system

Efficient typesetting and proofreading procedure

Display of the result of downloads and visits, as well as the number of cited articles Maximum dissemination of your research work

Submit your manuscript at: http://papersubmission.scirp.org/

Or contact ijmpcero@scirp.org 